\title{
MORTALIDADE INFANTIL EM MUNICÍPIO DO INTERIOR DO ESTADO DE SAO PAULO
}

\author{
INFANTY MORTALITY IN A COUNTY OF THE HINTERLAND \\ OF SAO PAULO STATE
}

\section{MORTALIDAD INFANTIL EN EL MUNICIPIO DEL INTERIOR DEL ESTADO DE SAO PAULO}

Kátia Poles*

Cristina Maria Garcia de Lima Parada**

Poles K, Parada CMGL- Mortalidade infantil em município do interior do Estado de São Paulo. Rev Esc Enferm USP $2002 ; 36(1): 10-7$.

\section{RESUMO}

Considerando que a mortalidade infantil é indicador dos niveis de saúde da população, realizamos este trabalho, cujo objetivo foi identificar as causas de mortalidade infantil no ano de 1998 em Botucatu. O coeficiente de mortalidade infantil obtido foi 12/ $1000 \mathrm{NV}$, com maior participação dos óbitos neonatais - 8,3/1000 NV A maior parte dos óbitos foi classificada como reduzivel ou parcialmente reduzivel, mas a atenção necessária para viabilizar tal redução foi variada. Dos óbitos ocorridos, 21,7\% eram inevitáveis, evidenciando que para redução dos índices de mortalidade infantil deveremos continuar investindo na qualidade da assistência à saúde e melhoria das condições de vida da população.

PALAVRAS -CHAVE: Mortalidade. Mortalidade infantil. Mortalidade neonatal. Mortalidade pós-neonatal.

\begin{abstract}
Considering that infant mortality indicates the levels of health in the population, we have accomplished the foolwing work, which goals were to identify the causes of infanty mortality during the year of 1998 in Botucatu. The rate of infanty mortality obtained was as much as 12/1000born alive with greater participation of the neonatal deaths - 8,311000 born alive. Though most of dealths can be classified as reducible or partially reducible, but the necessary attentionto make such reduction possible has varied. All the deaths that have occurred, 21,7\% were classified as unavoidable, emphasizing that to reduce the rates of infant mortality, we must continue investing on the quality of health assistance as well as on the improvement of life conditions of the population.
\end{abstract}

KEYWORDS: Mortality. Infant mortality. Neonatal mortality. Post-neonatal mortality.

\section{RESUMEN}

Considerando que la mortalidad infantil es indicador de los niveles de salud de la población, hemos realizado este trabajo, cuyo objetivo fue identificar las causas de mortalidad infantil en el año de 1998 en Botucatu. El coeficiente de mortalidad infantil obtenido fue 12/1000 NV, con mayor participación de los óbitos neonatales - 8,3/1000 NV. La mayor parte de los óbitos fue clarificada como reductible o parcialmente reductible, pero la atención necesaria para viabilizar tal reducción fue variada. De los óbitos ocurridos, el 21,7\% Bran inevitables, evidenciando que para reducción de los indices de mortalidad infantil deberemos seguir invirtiendo en la calidad de la asistencia a la salud y mejora de las condiciones de vida de la población.

PALABRAS -CLAVE: Mortalidad.Mortalidad Infantil. Mortalidad Neonatal.Mortalidad Post-Neonatal.

\footnotetext{
Enfermeira. Bolsista de Iniciação Científica CNPq durante o Curso de Graduação em Enfermagem. Kpoles@zipmail.com.br ** Professor Assistente Doutor do Departamento de Enfermagem da Faculdade de Medicina de Botucatu - UNESP. cparada@uol.com.br
} 


\section{INTRODUÇÃO}

O coeficiente de mortalidade infantil é indicador que, além de informar sobre os níveis de saúde de uma população, sintetiza as condições de bem-estar social, político e ético de uma dada sociedade. Significa a probabilidade de sobrevivência no primeiro ano de vida e, por isso mesmo, reflete não só as condições concretas de moradia, salário, alimentação e atenção à saúde, mas também, e talvez principalmente, o compromisso de uma sociedade com a reprodução social(1-2).

No primeiro ano de vida, os fatores biológicos e sociais têm grande influência sobre as condições de saúde das crianças, pois estas ainda estão em processo de formação e têm menor capacidade de defesa contra as agressões externas, favorecendo o desenvolvimento de doenças e a morte(3). Assim, estimativas da mortalidade infantil constituem um dos principais indicadores utilizados por organismos internacionais para avaliar o grau de desenvolvimento alcançado pelos países ${ }^{(4)}$.

Pode-se dizer que a mortalidade infantil é baixa quando inferior a 20 por mil(5). Atualmente, o Japão e a Suécia apresentam as menores taxas encontradas no mundo, com valores de 5 e 6 por mil, respectivamente. Na América do Sul, os países que apresentam as menores taxas de mortalidade infantil são o Chile e o Uruguai, com taxas de 15 e 19 por mil, respectivamente. O Brasil, no início da década de 90, apresentava mortalidade infantil de 54 óbitos por mil nascidos vivos(3).

Para avaliação da mortalidade infantil, esta tem sido dividida em dois componentes: mortalidade neonatal ou infantil precoce - que compreende os casos de crianças falecidas durante os primeiros 28 dias de vida - e mortalidade pós-neonatal ou infantil tardia que corresponde aos óbitos ocorridos entre o $29^{\circ}$ dia de vida e um ano de idade(5).

Esta divisão é feita para se avaliar as causas "endógenas", prevalentes no período neonatal, relacionadas às condições desfavoráveis do recémnascido, tais como imaturidade e anomalias congênitas e as causas "exógenas", com maior incidência no período pós-neonatal, produtos da atuação das condições ambientais sobre as crianças ${ }^{(1)}$

Estudo sobre a mortalidade infantil realizado em Botucatu-SP, evidenciou que $60,5 \%$ dos óbitos ocorreram no período neonatal e $39,5 \%$ no período pós-neonatal, pondo em evidência os períodos pré e perinatal como principais geradores de risco aos óbitos infantis. Assim, a participação dos riscos ligados às condições de vida, os quais têm relação direta com a mortalidade infantil tardia, foi menor(6).

Quando o coeficiente de mortalidade infantil é baixo, ele é quase totalmente representado pela mortalidade neonatal (60 a $80 \%$ ). Quando tal coeficiente é alto, observa-se o inverso, ele é quase totalmente representado pela mortalidade infantil tardia ou pós-neonatal(5)
Com o intuito de diminuir a mortalidade infantil, especialmente seu componente pós-neonatal, à partir da década de 80 foram realizadas grandes campanhas educativas para incentivar a amamentação e a utilização da terapia de reidratação oral contra a desidratação causada pelas diarréias ${ }^{(3)}$.

A expansão da rede de saneamento básico também contribuiu para a redução expressiva das mortes por doenças infecciosas intestinais, devido a associação entre óbitos gerados por doenças infecciosas e parasitárias e as condições de saneamento(6)

Vale ressaltar o decréscimo dos óbitos infantis tardios por doenças imunopreveniveis, desencadeado pela implementação do Programa Nacional de Imunizações nos anos 80 , pois mais crianças passaram a ficar protegidas contra doenças potencialmente mortais. Também deve ser destacado que, como a redução dos óbitos infantis decorrentes de malformações congênitas depende quase sempre de tecnologia avançada, essa é a parcela da mortalidade infantil que menos tem se alterado no mundo(3).

Se a tendência da mortalidade infantil é decrescente, mesmo em momentos de agravamento da crise sócio-econômica, deve-se considerar a importância das ações de saúde, tais como o controle das doenças imunizáveis. Por outro lado, a persistência de óbitos por causas evitáveis, como a diarréia e a pneumonia, nos grupos menos favorecidos, comprova a existência do problema em determinadas categorias sociais, bem como a omissão dos serviços de saúde em sua resolução (7)

$\mathrm{Na}$ atual política de saúde, voltada para a implementação do Sistema Único de Saúde (SUS), é conferida aos municípios a responsabilidade de gerir seu plano municipal de saúde. Neste sentido, a informação é concebida como suporte fundamental para esta gerência, seja no planejamento, gestão, organização ou na avaliação dos vários níveis de assistência(8). O exame das causas de óbito tem sido importante no planejamento de estratégias visando reduzir a mortalidade infantil. Dados locais são também necessários para conscientizar a população e dar apoio a programas de intervenção(9).

Partindo dessas afirmações é que nos propusemos a realizar este estudo, cujo objetivo foi identificar as causas de mortalidade infantil no ano de $1998 \mathrm{em}$ Botucatu-SP com a finalidade de fornecer subsídios aos serviços de saúde do município para o planejamento de suas atividades relativas às crianças menores de um ano.

\section{MATERIAL E MÉTODO}

\section{Local de realização do estudo}

O local onde se desenvolveu o presente trabalho é o município de Botucatu - SP Este localiza-se na 
região centro-sul do estado de São Paulo e abrange, além da sede, os distritos de Vitoriana e Rubião Júnior, Anhumas e Rio Bonito. Dista, aproximadamente, 230 $\mathrm{km}$ da capital, ocupa uma área de $1522 \mathrm{~km}^{2}$ e possuía, no ano de 1998, uma população estimada de 105.230 habitantes.

A rede básica dos Serviços de Saúde de Botucatu tem como objetivo promover, prevenir, diagnosticar e reabilitar com cobertura universal, sem distinção entre clientela previdenciária e não previdenciária, tentando resolver em nível local os principais problemas de saúde da população e articulando-se com os serviços públicos de nível secundário e terciário para o atendimento especializado(10). Estudos como este visam contribuir para que a rede básica de saúde aproxime-se deste seu objetivo.

\section{Tipo de Estudo}

Trata-se de estudo descritivo e as variáveis estudas, apresentadas através de medidas de frequência, agrupadas em 6 itens:

- caracterização das mães: idade, grau de instrução e situação conjugal;

- perfil sócio-econômico familiar: ocupação das mães, renda familiar e condições de moradia;

- história obstétrica pregressa das mães e assistência pré-natal: paridade, intervalo interpartal, época de início do pré-natal, número de consultas, exames realizados e intercorrências na gravidez;

- assistência ao parto: tipo de parto, local de realização, profissional que realizou a assistência;

- condiçães de nascimento e evolução dos bebês: sexo, peso e idade gestacional ao nascer, índice de Apgar, profissional que realizou a assistência e local de internação;

- descrição do óbito: época de ocorrência e causa básica de morte segundo critérios de evitabilidade.

\section{Coleta de dados}

Foram estudados todos os óbitos infantis ocorridos no município de Botucatu-SP no ano de 1998. Como fonte de dados utilizamos as informações contidas no atestado de óbito e declaração de nascidos vivos, que foram fornecidas pelo Núcleo de Ação Coletiva do Centro de Saúde Escola da Faculdade de Medicina de Botucatu, bem como informações dos prontuários da criança e sua mãe.

Além disso, realizamos entrevistas domiciliárias com as mães das crianças ou, na ausência destas, parentes próximos que, na época do óbito, residiam com a criança, com a finalidade de obtermos maiores informações.

Para consultarmos os prontuários, obtivemos autorização da Secretaria Municipal de Saúde do Município, do diretor do Centro de Saúde Escola e dos diretores clínicos dos hospitais envolvidos.

Desta forma, obtivemos informações detalhadas sobre os períodos pré-natal, neonatal e pós-neonatal, as quais nos permitiram elucidar toda a história pregressa ao óbito, assim como relacionar os fatores que tinham ligação com a mortalidade infantil.

Foi preservado o anonimato dos serviços de saúde e das mães envolvidas no estudo. Vale ressaltar que este trabalho foi avaliado a aprovado por Comitê de Ética em Pesquisa.

\section{RESULTADOS E DISCUSSÃO}

Durante o ano de 1998 ocorreram 23 óbitos infantis no município de Botucatu-SP. Destes, 16 ocorreram no período neonatal e 7 no período pósneonatal. Assim, observamos a participação do componente neonatal e pós-neonatal de 69,6\% e 30,4\%, respectivamente, na mortalidade infantil, o que evidencia um acréscimo na proporção de óbitos neonatais, quando comparamos estes dados com aqueles obtidos em estudo realizado no mesmo município em $1987^{(6)}$. Considerando que o número de nascidos vivos no mesmo ano foi de 1912, o coeficiente de mortalidade infantil encontrado foi de 12 por mil nascidos vivos, valor que pode ser considerado baixo(5). É, então, compreensível, a maior participação proporcional dos óbitos neonatais - 8,3 óbitos por mil nascidos vivos - e menor participação do componente pós-neonatal - 3,7 óbitos por mil nascidos vivos.

\section{Caracterização das mães}

A idade materna tem sido apontada como fator de risco para a mortalidade infantil, pois a gestação nos extremos da vida reprodutiva: abaixo de 20 e acima de 35 anos, pode trazer risco tanto para mãe quanto para o bebê ${ }^{(11)}$. Em nosso estudo, a idade das 23 mães variou de 14 a 43 anos, sendo que 14 delas eram adolescentes, pois tinham menos de 20 anos no momento do parto. Ponderamos que nessa fase da vida, um filho quase sempre traz problemas sociais e econômicos, principalmente para a mulher. Apenas 2 mães tinham idade igual ou superior a 35 anos.

Quanto ao grau de instrução, a maioria das mães (14 casos) não havia completado o ensino fundamental, 7 mulheres haviam concluído o ensino médio e as restantes ( 2 casos) haviam completado o ensino fundamental, tendo iniciado o ensino médio.

O aumento da escolaridade da população, em especial das mulheres, é uma das formas mais eficientes de evitar o risco de mortalidade infantil, pois, de maneira geral, as mulheres com mais tempo de 
estudo são mais capazes de regular sua fecundidade e mais sensiveis às campanhas de educação em saúde, aderindo mais facilmente a programas de puericultura $(3,6)$

Levando em consideração que a estabilidade conjugal pode traduzir maiores chances de seguridade social para a criança, é importante destacarmos que embora 5 mulheres não tivessem companheiro, as demais eram casadas ou tinham união estável.

\section{O perfil sócio-econômico familiar}

Estudamos o perfil sócio-econômico familiar porque altos indices de mortalidade também relacionam-se com baixa renda: nota-se relação entre renda familiar, risco de desnutrição e menor consumo de bens e serviços (12)

Com relação a ocupação das mães, 8 delas exerciam atividade remunerada no momento do parto e trabalhavam fora de casa o dia todo, diariamente.

A renda familiar foi bastante variada e para melhor estudá-la, utilizamos a renda per capta familiar (RPCF), que variou de 0,1 a 19,2 salários minimos. Assim, 8 famílias foram consideradas não pobres, pois possuiam RPOF superior a 1,8 salários minimos; 7 familias foram consideradas pobres, com RPCF entre 0,6 a 1,8 salários mínimos e 8 familias foram consideradas miseráveis, com RPCF inferior a 0,6 salário minimo(13).

É importante destacarmos que entre os casos de óbitos pós-neonatais, os quais têm estreita relação com as condições de vida da população, todas as familias foram consideradas pobres ou miseráveis, pois tinham RPCF inferior a 1,8 salários minimos.

A importância da renda na determinação do processo saúde-doença é consenso entre os autores, pois este é um indicador através do qual os agentes, de determinada classe social, conseguem consumir bens e serviços, como assistência médica, escola, alimentos, entre outros, essenciais para a manutenção da saúde (14)

Das 23 famílias, 11 moravam em casa própria, 6 em casa cedida e as outras 6 pagavam aluguel. Em apenas 1 casa o abastecimento de água era através de poço e o lixo incinerado; em 2 delas, havia fossa para destino dos dejetos.

Embora as crianças com inadequação no abastecimento de água apresentem um risco de morrer 8,5 vezes maior que as outras (6), este não parece ter sido um fator importante neste estudo, já que apenas uma casa não dispunha de água tratada, sugerindo que os óbitos pós neonatais decorrentes desse fator podem já estar controlados.

\section{História obstétrica pregressa das mães e assistência pré-natal}

Em estudos acerca da mortalidade infantil fazse necessário conhecer a história obstétrica pregressa das mães, pois sabe-se que a mortalidade é maior no caso de mães que tiveram mais gestações, isto é, nas grandes multiparas e quando o intervalo interpartal é de um ano ou menos $(12,15)$

Com relação ao número de gestações, em nosso estudo, 9 mulheres eram primigestas, sendo que o número máximo de gestações observado foi 5 (2 casos); houve 8 casos de aborto anterior e nenhum caso de natimortalidade. Estes casos de aborto foram observados entre as mães dos bebês que foram a óbito no período neonatal, evidenciando a necessidade de se aprofundar os estudos acerca de aborto anterior como fator de risco ao óbito nos primeiros 28 dias de vida. Por ora, parece-nos que mulheres com história de aborto anterior devam receber cuidadosa atenção pré-natal, com vistas a evitar, em suas gestações, o parto prematuro e, consequentemente, reduzir os indices de mortalidade, especialmente a neonatal.

Quanto ao intervalo interpartal, das 14 mulheres multiparas, apenas 4 tinham intervalo igual ou inferior a um ano. Tendo em vista o pequeno número de mulheres que atendiam a esta condição, este estudo não pode aprofundar a discussão acerca da relação entre pequeno intervalo interpartal e mortalidade infantil.

Também é importante, em investigações sobre mortalidade infantil, estudar a assistência pré-natal: é sabido que problemas de saúde da mãe quase sempre repercutem na saúde do feto $\mathrm{e}$, na medida que o acompanhamento pré-natal permite identificar algumas doenças e prevenir outras, acaba beneficiando a saúde da mãe e do filho ${ }^{(3,16)}$ Foi possivel obter informações sobre o pré-natal em 22 casos. Destas mães, todas frequentaram serviço de assistência pré-natal, sendo que a maioria (16 casos) utilizou serviço público de saúde e as restantes (6 casos) utilizaram serviço particular.

Considera-se adequado o início dos cuidados prénatais no primeiro trimestre de gravidez ${ }^{(11,17)}$ e quase dois terços das mães estudadas (15 casos) estavam nesta situação, pois haviam iniciado o pré-natal até o terceiro mês de gestação. Com relação ao número de consultas pré-natais, 9 mulheres compareceram a pelo menos 6 consultas, número mínimo considerado para uma assistência pré-natal efetiva. Entretanto, devemos ressaltar que 14 partos ocorreram prematuramente e, como o número de consultas pré-natais aumenta com a proximidade do termo, este pode ter sido o motivo pelo qual mais da metade das gestantes estudadas compareceu a menos de 6 consultas pré-natais.

Todas as mulheres fizeram os exames de sangue e urina preconizados na rotina do atendimento prénatal e a maioria (14 casos) realizou ultrassonografia obstétrica em algum momento antes do parto.

Assim, embora todas as mães tenham recebido assistência pré-natal, realizado os exames de rotina preconizados e a maior parte delas ter iniciado este 
acompanhamento precocemente, não foi possivel evitar o óbito. Entretanto, deve-se ponderar que por ser o pré-natal uma atividade rotineira, sujeita a simplificações nem sempre desejáveis, a qualidade com que é realizado pode ser o fator determinante para sua efetividade, daí a necessidade de se realizar estudos que avaliem aspectos qualitativos desta forma de assistência.

Mais de dois terços das mulheres não apresentou problemas de saúde durante a gravidez (16 casos) e o problema com maior frequência encontrado foi o trabalho de parto prematuro (4 casos).

Foram internadas 7 gestantes, sendo o motivo que levou a internação, com maior frequência, o trabalho de parto prematuro (6 casos) e a outra mulher que necessitou, internou-se para controle de pressão arterial.

\section{Assistência ao parto}

A respeito da assistência ao parto, há estreita relação entre a qualidade com que é prestada e a mortalidade infantil precoce, pois problemas decorrentes deste, quando realizado com técnicas inadequadas, têm repercussão sobre o recém-nascido durante os primeiros dias de vida(18).

Embora a cobertura da assistência pré-natal tenha sido alta, 7 mulheres não tinham, no momento de internação para o parto, qualquer carta de encaminhamento ou cartão de pré-natal com informações sobre a gestação, que pudessem subsidiar o atendimento hospitalar, pois embora tivessem recebido tal documento durante a gravidez, não sabiam da importância de levá-lo no momento do parto.

$\mathrm{O}$ parto foi frequentemente realizado por médico plantonista: 18 casos; 4 mulheres tiveram seus partos realizados pelo profissional que as acompanhou no pré-natal e uma delas deu a luz antes de chegar ao hospital, sendo atendida por auxiliar de enfermagem.

Com relação ao tipo de parto, estudos apontam para maior mortalidade entre bebês nascidos de partos cirúrgicos, quando comparados aos nascidos de parto normal (2-3) Dos 23 partos estudados, 19 foram vaginais e 4 cesárea, assim, a incidência de cesárea aqui observada está pouco acima daquela preconizada pela Organização Mundial de Saúde (OMS) para população em geral: 10,0 a $15,0 \%{ }^{(19)}$.

Quanto ao local do parto, 8 ocorreram em hospital secundário e 15 em hospital terciário. Os partos realizados em hospital terciário o foram, em geral, devido a problemas apresentados durante a gestação, como o trabalho de parto prematuro.

Dos 16 óbitos neonatais, 13 ocorreram em hospital terciário e entre os 7 óbitos pós-neonatais, o local mais frequente de nascimento foi o hospital secundário.

\section{Condições de nascimento e evolução dos bebês}

A grande maioria dos bebês (15 casos) foi assistida por pediatra no momento do parto, 3 bebês receberam assistência de profissional da área de enfermagem e 5 mães não souberam referir quem assistiu a seus filhos, também não sendo possivel identificar o profissional através do prontuário hospitalar.

Das 23 crianças, 11 eram do sexo feminino e 12 do sexo masculino e as condições de nascimento elucidaremos a seguir. O peso ao nascimento e a idade gestacional são apresentados nas Tabelas 1 e 2.

Tabela 1 - Peso ao nascer (g), das crianças que foram a óbito no primeiro ano de vida. Botucatu, 1998.

\begin{tabular}{ccc}
\hline Peso (g) & $\begin{array}{c}\text { Óbitos } \\
\text { Neonatais }\end{array}$ & $\begin{array}{c}\text { Óbitos pós- } \\
\text { neonatais }\end{array}$ \\
\cline { 2 - 3 } & $\mathbf{N}$ & $\mathbf{N}$ \\
\hline $475 \mid--1000$ & 8 & - \\
$1000 \mid--1500$ & 2 & 1 \\
$1500 \mid--2500$ & 3 & 2 \\
$2500 \mid--3800$ & 3 & 4 \\
\hline Total & 16 & 7 \\
\hline
\end{tabular}

Tabela 2 - Idade gestacional (IG) em semanas, ao nascimento, das crianças que foram a óbito no primeiro ano de vida. Botucatu, 1998.

\begin{tabular}{ccc}
\hline \multirow{2}{*}{ IG (semanas) } & $\begin{array}{c}\text { Óbitos } \\
\text { neonatais }\end{array}$ & $\begin{array}{c}\text { pós-neonatai } \\
\text { Óbitos }\end{array}$ \\
\cline { 2 - 3 } & $\mathbf{N}$ & $\mathbf{N}$ \\
\hline $22|-| 127$ & 9 & - \\
$28|-| 36$ & 4 & 1 \\
$37|-| 41$ & 3 & 6 \\
\hline Total & 16 & 7
\end{tabular}

É indiscutivel a importância da prematuridade para a mortalidade neonatal, seja devido a imaturidade pulmonar, seja devido à falta de defesas(6). Também o recém-nascido com baixo peso ao nascer - abaixo de 2500 $\mathrm{g}$ - apresenta risco aumentado de mortalidade(20), pois a variável peso apresenta estreita relação com a idade gestacional, sendo a mortalidade proporcio-nalmente maior, quanto menor a idade gestacional e o peso (21).

Analisando as Tabelas 1 e 2, podemos observar a clara relação entre peso e idade gestacional. Entre os bebês que foram a óbito no período neonatal, notase que 3 deles, com peso adequado ao nascimento, eram os mesmos com idade gestacional acima de 37 semanas e os outros, com peso ao nascer inferior a $2500 \mathrm{~g}$, tinham algum grau de prematuridade. Para o grupo dos óbitos tardios é possível dizer que o peso e a idade 
gestacional não foram fatores marcantes de risco ao óbito, pois nenhum bebê tinha prematuridade extrema e/ou muitíssimo baixo peso. Assim, fica claro que aqueles bebês, portadores destes dois fatores de risco, são mais suscetiveis ao óbito nos primeiros 28 dias de vida, evidenciando, novamente, a importância das causas perinatais nos óbitos neonatais.
Na Tabela 3 apresentamos os indices de Apgar das crianças. Cabe aqui ressaltar que o índice de Apgar no primeiro minuto de vida geralmente relaciona-se com o pH do cordão umbilical e traduz uma asfixia intraparto, sendo que a nota do quinto minuto correlaciona-se com eventuais sequelas neurológicas (22)

Tabela 3 - Índice de Apgar no primeiro e quinto minutos de vida das crianças que foram a óbito no primeiro ano de vida. Botucatu, 1998.

\begin{tabular}{ccccc}
\hline & \multicolumn{2}{c}{$\mathbf{1}^{\circ}$ minuto de vida } & \multicolumn{2}{c}{$\mathbf{5}^{\circ}$ minuto de vida } \\
\hline \multirow{2}{*}{ Apgar } & Óbitos neonatais & $\begin{array}{c}\text { Óbitos } \\
\text { pós-neonatais }\end{array}$ & $\begin{array}{c}\text { Óbitos neonatais } \\
\text { Óbtos } \\
\text { pós-neonatais }\end{array}$ \\
\cline { 2 - 5 } & $\mathbf{N}$ & $\mathbf{N}$ & $\mathbf{N}$ & - \\
\hline $0|-| 3$ & 14 & - & 4 & - \\
$4|-| 6$ & 1 & 1 & 8 & - \\
7 & - & - & 1 & 7 \\
$81-110$ & - & 6 & 3 & - \\
não consta* & 1 & - & - & 7 \\
\hline Total & 16 & 7 & 16 & \\
\hline
\end{tabular}

:* Nasceu na ambulância, recebeu índice de Apgar apenas no $5^{\circ}$ minuto de vida, após chegar ao Hospital para o qual estava sendo encaminhada a gestante

Na Tabela 3 podemos observar que dos 16 bebês que foram a óbito no período neonatal, 15 tiveram anóxia intra parto grave ou moderada, pois apresentaram, no primeiro minuto após o nascimento, índice de Apgar entre 0 e 6 . A recuperação foi boa para um quarto dos bebês, que atingiram nota 7 ou superior. Entre os óbitos tardios, apenas 1 bebê apresentou depressão grave no primeiro minuto, com boa recuperação no quinto minuto e tal bebê apresentava anomalia congênita. Estes dados confirmam que o periodo neonatal é crítico para aqueles bebês que apresentam, já nos primeiros minutos de vida, algum grau de depressão.

Entre os bebês que foram a óbito no período neonatal, o local mais frequente de internação foi a UTI Neonatal (13 casos) e as 7 crianças que foram a óbito no período pós-neonatal ficaram, com maior frequência, no alojamento conjunto ou berçário normal (5 casos).

\section{Descrição do óbito}

Dos 16 bebês que faleceram no periodo neonatal, metade ocorreu no primeiro dia de vida e $87,5 \%$ no período neonatal precoce, ou seja, nos primeiros sete dias de vida, e tinham, na maioria dos casos, algum grau de prematuridade e/ou comprometimento logo ao nascer. Já entre os óbitos tardios, os quais estão mais relacionados às condições ambientais desfavoráveis, ocorreram, em geral, após 2 meses de vida.

Apontaremos, nos Quadros 1 e 2, a causa básica de morte, definida como doença (ou lesões) que iniciou a cadeia de acontecimentos patológicos que conduziram diretamente à morte e/ou circunstâncias do acidente ou violência que produziram a lesão fatal, segundo critérios de evitabilidade(23). 
Quadro 1 - Causas básicas de morte dos bebês que foram a óbito no período neonatal, segundo critérios de evitabilidade. Botucatu, 1998.

\begin{tabular}{|l|l|c|}
\hline \multicolumn{1}{|c|}{ Classificação } & \multicolumn{1}{c|}{ Causa básica de morte } & N \\
\hline Reduzivel por adequada atenção ao parto & Materna indeterminada / anóxia & 1 \\
\hline \multirow{2}{*}{ Aspiracão meconial } & 1 \\
\hline Parcialment por diagnóstico e tratamento precoces & Infecção adquirida & 3 \\
gravidez & Amniorexe prematura & 2 \\
& Incompetência istmo-cervical & 3 \\
\hline \multirow{2}{*}{ Não evitável } & Sindrome da angústia respiratótia & 1 \\
\hline & Materna indeterminada / prematuridade & 2 \\
\hline
\end{tabular}

Quadro 2 - Causas básicas de morte das crianças que foram a óbito no período pós-neonatal, segundo os critérios de evitabilidade. Botucatu, 1998.

\begin{tabular}{|l|l|c|}
\hline \multicolumn{1}{|c|}{ Classificação } & \multicolumn{1}{|c|}{ Causa básica de morte } & N \\
\hline Reduzivel por diagnóstico e tratamento precoces & Broncopneumonia & 2 \\
\cline { 2 - 3 } & & 1 \\
Parcialmente reduzivel por diagnóstico e & Asfixia mecânica por sufocação & 1 \\
tratamento precoces & Sindrome da criança espancada & 1 \\
\hline Nesnutrição & 1 \\
\hline
\end{tabular}

Analisando os Quadros 1 e 2, podemos observar que, dos 23 óbitos ocorridos em Botucatu - SP no ano de 1998, apenas 5 podem ser classificados como óbitos não evitáveis. Entretanto, foram marcantes as diferenças de causas básicas de morte para os 2 grupos de óbitos. Assim, entre os 16 óbitos neonatais, 11 podem ser classificados como reduziveis por adequada atenção ao parto ou parcialmente reduziveis por adequado controle da gestação. Quanto aos 7 óbitos pós-neonatais, 5 podem ser classificados como reduziveis ou parcialmente reduziveis por diagnóstico e tratamento precoces.

\section{CONSIDERAÇÕES FINAIS}

As assistências pré-natal e pós-natal devem permitir a identificação de gestantes e recém-nascidos de risco, para que os mesmos recebam pronta e competente assistência. Isto poderia evitar óbitos decorrentes de causas evitáveis.

No caso específico deste estudo, mais de dois terços dos óbitos poderiam ter sido total ou parcialmente evitados e, por tratar-se de óbitos infantis, isso só ocorrerá com a melhoria não apenas da qualidade da assistência à saúde prestada nos diferentes niveis de complexidade às crianças, mas também com a melhoria das condições de vida - educação, moradia, alimentação, salário, entre outros - de toda população, especialmente da parcela mais carente.

Quanto a assistência à saúde, a busca pela qualidade poderá passar por:

- Formulação de projetos precedidos de estudos técnicos, a fim de se verificar as prioridades locais;

- Investimentos em tecnologias conhecidas, que possam ser utilizadas nos casos de óbitos evitáveis;

- Considerar os fatores de risco ao óbito infantil desde o atendimento pré-natal, como a renda, escolaridade, fatores maternos, entre outros;

- Detectar e acompanhar adequadamente as gestações de alto risco no pré-natal;

- Utilizar mecanismos eficientes de referência e contra-referência entre os centros de saúde, maternidades e hospitais infantis.

Agradecimentos: Às mães, que prontamente contribuíram para o êxito deste trabalho. 


\section{REFERÊNCIAS BIBLIOGRÁFICAS}

(1) Ferreira CEC, Monteiro CA, Zuñiga HPP. A mortalidade. In: Monteiro CA. Saúde e nutrição das crianças de São Paulo. São Paulo: Hucitec Nupens/USP; 1988.

(2) Leal MC, Szwarcwald CL. Evolução da mortalidade neonatal, no estado do Rio de Janeiro, Brasil, de 1979 a 1993. 1. Análise por grupo etário segundo região de residência. Rev Saúde Públ 1996; 30: 403-12.

(3) Telarolli Jr R. Mortalidade infantil: uma questão de saúde pública. São Paulo: Moderna; 1997.

(4) Monteiro CA, Nazário CL. Declínio da mortalidade infantil e eqüidade social: o caso da cidade de São Paulo. In: Monteiro CA. Velhos e novos males de saúde no Brasil. São Paulo: Hucitec Nupens/ USP; 1995.

(5) Laurenti R, Jorge MHPM, Lebrão ML, Gotlieb SLD. Estatísticas de saúde. São Paulo: Editora Paulista e Universitária; 1987.

(6) Macharelli CA, Oliveira LR. Perfil do risco de óbito de crianças menores de um ano residentes em localidade do estado de São Paulo, Brasil, 1987. Rev Saúde Publ 1991; 25: 121-8.

(7) Hartz ZMA. Mortalidade infantil "evitável" em duas cidades do nordeste do Brasil: indicador de qualidade do sistema local de Saúde. Rev Saúde Públ 1996; 30: 310-18-

(8) Souza RKT, Soares DFPP, Fonzar UJV. Mudanças demográficas e perfil de mortalidade no municipio de Maringá-PR, entre 1980 e 1991. Saúde Debate 1996; 51: 7581-

(9) Nakamura RM. Excess infant mortality in an American Indian Population, 1940-1990. J Am Méd Assoc 1991; 266: 2244-48.

(10)Botucatu. Prefeitura Municipal- Centro de Saúde Escola. Escritório Regional de Saúde. Plano diretor de saúde. Botucatu 1992. / Mimeografado/.

(11) Gomes JO, Santo AH. Mortalidade infantil em município da região centro-oeste paulista, Brasil, 1990 a 1992. Rev Saúde Públ 1997; 31: 330-41.

(12) Paim JS, Costa MCN. Decréscimo e desigualdade da mortalidade infantil: Salvador, 1980-1988. Bol Oficina Sanit Panam 1993; 114: 415-28-

(13) Fundação SEADE. Pobreza e riqueza: pesquisa de condições de vida na região metropolitana de São Paulo. São Paulo: SEADE; 1992.

(14) Macharelli CA. Mortalidade perinatal em Botucatu, SP, 1991/1992. (Mestrado), Ribeirão Preto(SP): Departamento de Medicina Social da Universidade de São Paulo; 1995.

(15) Sherman AJ. Mortalidade infantil e sobrevida de crianças na Bahia, Brasil: uma revisão da literatura sócioepidemiológica. Rev Saúde Públ 1989; 16: 15-29.
(16) Barbieri MA, Zucoloto S, Gomes VA, Gurgel RQ Cipolotti R, Kajwiara JK, Oba LH, Silva OJMF, Wen LI. Estudo do comportamento das taxas de mortalidade fetal, perinatal e neonatal em um hospital universitário. $J$ Pediatr 1988; 64: 205-10.

(17) Grandi C, Saraquesta P. Crontol prenatal: evaluación de los requisitos básicos recomendados para diminuir ei dano perinatal- J Pediatr 1997; 73: 15-20.

(18) Barros FC, Victora CG, Vaughan JP. Causas de mortalidade perinatal em Pelotas, RS (Brasil)- Utilização de uma classificação simplificada. Rev Saúde Públ 1987; 21: 310-16.

(19)Organização Mundial de Saúde (OMS)- Appropriate technology for birth. Lancet 1985; 2: 436-37.

(20) Barros Filho AA, Mariotoni GGB, Wacked WB. O recém-nascido de baixo peso: morbidade e mortalidade hospitalares, Maternidade de Campinas, 1994. Rev Paul Pediatr 1996; 14: 101-06.

(2 1) Calif VMLT. Caracterização do recém-nascido pré-termoIn: Leone CR, Tronchin DMR. Assistência integrada ao recém-nascido. São Paulo: Atheneu; 1996.

(22) Segre CAM, Marino WT. Atendimento ao RN na sala de parto- In: Segre CAM, Armellini PA, Marino WT. RN. São Paulo: Sarvier; 1995.

(23) Ortiz LP Utilização das causas evitáveis na mortalidade infantil como instrumento de avaliação das ações de saúde. In: Anais do $10^{\circ}$ Encontro Nacional de Estudos Populacionais; 1996. Caxambu; 1996. P.225368.

\section{Artigo recebido em 11/09/00}

\section{Artigo aprovado em 07/11/01}

